\title{
Quality and patient safety committee structure and activities in an academic department of anesthesiology: a narrative description
}

\section{Structure et activités d'un comité responsable de la qualité de l'acte et de la sécurité des patients dans un département d'anesthésiologie universitaire : une description narrative}

\author{
Christopher L. Pysyk, MD, FRCPC · Lucie Filteau, MD, FRCPC · Alan Baxter, BM, BS, MA, FRCA, FRCPC
}

Received: 26 February 2019/Revised: 9 July 2019/Accepted: 10 July 2019/Published online: 13 September 2019

(C) Canadian Anesthesiologists' Society 2019

\begin{abstract}
Quality and patient safety (QPS) activities continue to attract more attention and are deemed an essential component of care provision by all departments of anesthesiology, but examples of the structure and processes to manage QPS matters at the department level in Canada are not well described in the literature. This narrative article highlights the creation, structure, evolution, and experiences of a QPS committee in a Canadian department of anesthesiology and pain medicine. Specific focus and case examples of the QPS committee's use of a hospital-wide incident reporting system to monitor and respond to perioperative QPS matters are provided.
\end{abstract}

Résumé Les activités de gestion de la qualité de l'acte et de la sécurité des patients (QSP) continuent d'attirer de plus en plus d'attention et sont considérées comme une composante essentielle de la prestation de soins par tous les départements d'anesthésiologie, mais les exemples de la structure et des processus pour gérer les questions de QSP au niveau départemental au Canada ne sont pas bien décrits dans la littérature. Cet article narratif met en lumière la création, la structure, l'évolution et les expériences d'un comité responsable de la QSP dans un département d'anesthésiologie et de traitement de la

C. L. Pysyk, MD, FRCPC ( $₫)$ - L. Filteau, MD, FRCPC . A. Baxter, BM, BS, MA, FRCA, FRCPC

Department of Anesthesiology and Pain Medicine, Faculty of Medicine, University of Ottawa, Ottawa, ON, Canada

e-mail: cpysyk@toh.on.ca

Department of Anesthesiology and Pain Medicine, The Ottawa Hospital, General Campus, 501 Smyth Rd, Critical Care Wing 1401, Ottawa, ON K1H 8L6, Canada douleur canadien. Nous mettons une emphase particulière et présentons des exemples de scénario sur l'utilisation du comité de QSP d'un système de rapport d'incidents à l'échelle de l'hôpital afin de surveiller et de répondre aux questions de QSP périopératoires.

Various approaches to the management of quality and patient safety (QPS) activities in both perioperative and non-perioperative care paradigms have been described at individual, departmental, hospital, and health-system levels. ${ }^{1-4}$ Nevertheless, the scope and context of QPS activities conducted within a Canadian department of anesthesiology and pain medicine-particularly in a department with experience using a patient safety incident reporting system-have received little focus in the literature. Recent unpublished survey results reveal that not all Canadian academic departments of anesthesiology have a QPS committee, with those that do not having plans to establish one.

With recent changes to the Canadian Anesthesiologists' Society's (CAS) Guidelines to the Practice of Anesthesia, ${ }^{5}$ the requirement for all departments, regardless of size, to "employ a systematic approach for monitoring the quality of anesthetic care provided by members of the Department of Anesthesia throughout the healthcare facility" may be viewed by some as a daunting addition.

In this narrative review, we discuss key features of our (i.e., an academic, tertiary care department of anesthesiology and pain medicine) QPS committee's structure, design, functionality, and lessons learned, with particular focus on use of a hospital-wide incident 
reporting system. Using a case-based approach, we outline our experience designing and modifying our QPS committee. Given the indeterminate state of QPS activities in departments of anesthesiology across Canada, our experience may be of interest to those departments seeking an approach to the management of QPS matters and structure of a QPS committee.

\section{Setting and use of a reporting system to facilitate learning and incident review}

In 2011, all clinical departments at The Ottawa Hospital (TOH) were encouraged to create a structured process to review and manage quality of care and patient safety matters relevant to each department's clinical area of focus. The Department of Anesthesiology and Pain Medicine at TOH delivers clinical care for $>30,000$ inpatient and outpatient procedures each year across a multi-campus academic health sciences centre setting. The department consists of 83 full-time equivalent staff anesthesiologists, 68 residents/fellows, and 16 anesthesia assistant respiratory therapists (AART).

Use of the existing institution-wide incident reporting system (patient safety learning system [PSLS], Datix Ltd, London, UK), which had been recently introduced at TOH, became a central focus for QPS activities in each department at that time. The PSLS is a hospital-wide, electronic incident reporting platform accessible on $\mathrm{TOH}$ computers to all hospital employees, physicians, and trainees. The software uses free text and dropdown menus to characterize and direct the reports accordingly for review. The reports are reviewed by $\mathrm{TOH}$ employees/physicians with a QPS role in the department/clinical care area where the report originated. If needed, reviewers from other departments/care areas can be "actioned" or contacted through the PSLS platform to provide a multidisciplinary perspective of the event. The PSLS reviewers are notified by email that a report has been entered in their respective care area. The event reporter (i.e., person who entered the event) receives a notification email that the report has been directed for review and can access the PSLS to follow the progress of the report including any subsequent actions that are derived from the review. The event reporter's name is available to PSLS reviewers to facilitate further discussion of the event, if needed.

The current CAS Guidelines identify and encourage adverse event reporting systems as one way to monitor quality of anesthetic care throughout the healthcare facility. ${ }^{5}$ Review of the PSLS reports informs much of our QPS committee's focus and direction to identify, assess, and manage threats to patient safety at our centre.
Reporting patient safety events for review by a predetermined body of peers (such as members of a QPS committee) provides a local granular perspective and interpretation that national and international incident reporting databases may not be able to achieve. It is important, however, to juxtapose and compare one's local reports with the trends noted elsewhere for a balanced, complimentary view of safety matters in the perioperative domain. In addition, the local reporting system also generates an invaluable source of peer-vetted cases to aid the conduct of morbidity and mortality rounds, another feature of monitoring quality of anesthesia care identified in the Guidelines. ${ }^{5}$

For anesthesia departments without an established infrastructure for reporting and reviewing events, the recently launched Canadian Anesthetic Incident Reporting System (CAIRS) ${ }^{6}$ will hopefully foster and facilitate a reporting culture in perioperative medicine to improve systems of care. Like any reporting system, CAIRS is a tool that may provide feedback and information about events to support quality and safety improvement at the department, hospital, and health-system levels of care. Nevertheless, like any tool, its products and outputs should be interpreted, reviewed, and applied by the appropriate healthcare professionals who are knowledgeable about the intricacies of their own local care environment.

At our centre, when a report is entered into the PSLS, a predetermined group of departmental QPS clinical reviewers (who are staff anesthesiologists) receive an email notification that an event is available for review. In the PSLS report, the patient's medical chart number is listed, enabling the reviewer to access the patient's electronic medical record for further details of the event and care the patient received. The PSLS clinical reviewers from each $\mathrm{TOH}$ campus (two staff anesthesiologists from each inpatient campus and one staff anesthesiologist from the outpatient campus) evaluate the PSLS reports submitted from their respective site. Each case is then considered for presentation and discussion by the committee at the monthly QPS committee meeting. If system and/or cognitive issues are involved in the event, further discussion of the case is warranted at the committee level. Case specifics are further expanded upon as necessary by the clinical reviewer at the monthly QPS committee meeting. During discussion of the PSLS reports, the QPS committee determines next steps and/or actions (if appropriate) for the cases reviewed. Specifically, the QPS committee assesses if a threat to quality/patient safety exists and if remediable actions are feasible to prevent future occurrences. The mode of dissemination of the information learned from case review to perioperative colleagues is also considered. If there are system and/or cognitive issues that merit presentation of the report, the 


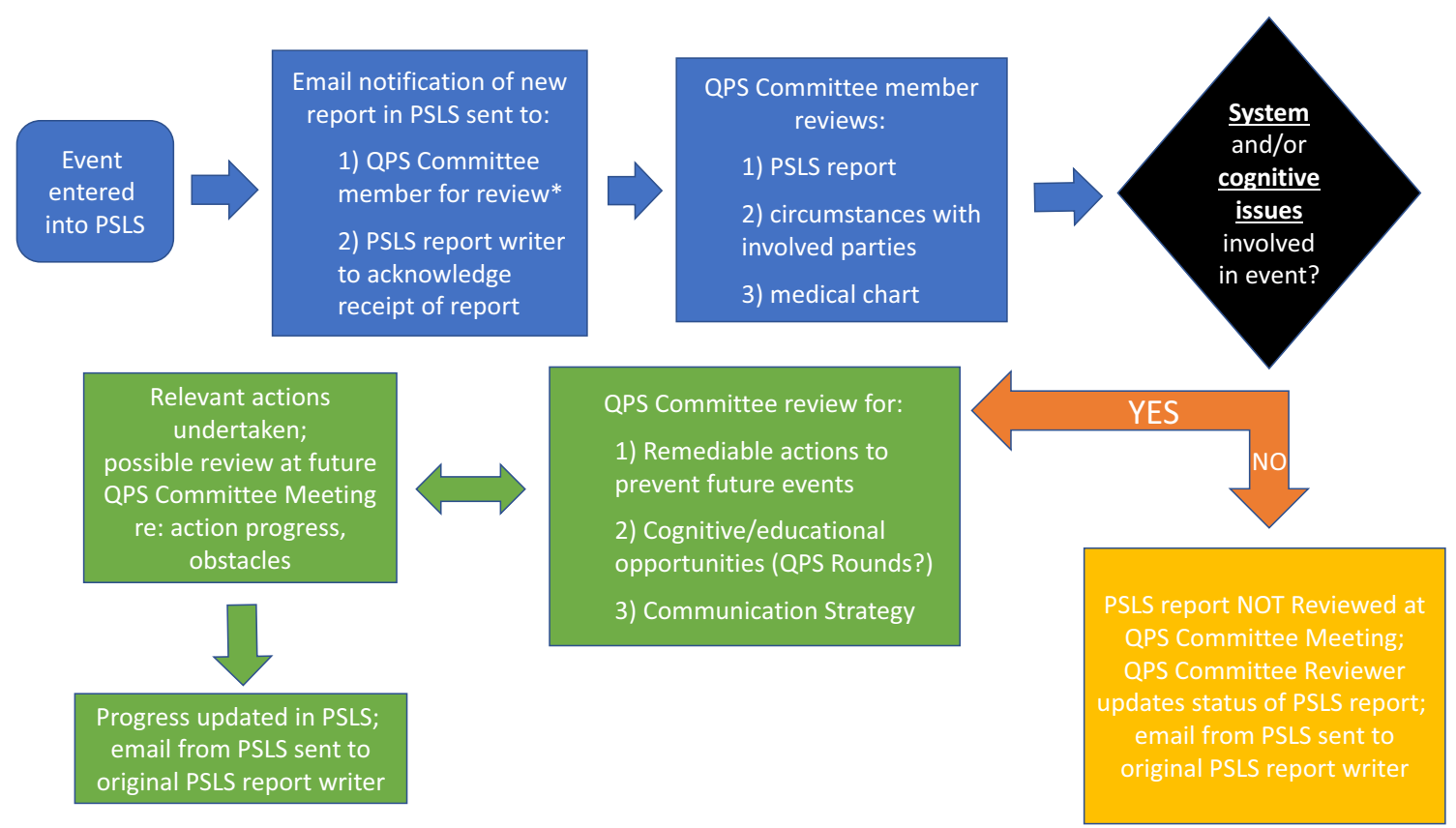

Fig. 1 Schematic flow diagram outlining quality and patient safety (QPS) committee process for management of patient safety learning system (PSLS) reports. *Potential site for reports submitted via

event is added to the list of possible cases for presentation at "QPS rounds" (departmental morbidity and mortality rounds).

The committee selects suitable PSLS cases for "QPS rounds", presented using the Ottawa Morbidity and Mortality Model (OM3) ${ }^{7}$ format, which occur every six to twelve weeks within our department's grand rounds schedule. An overview of PSLS report management via the QPS committee is provided in Fig. 1.

The QPS committee functionality is in keeping with institutional policies and Ontario provincial law ${ }^{8}$ surrounding collection, use, and disclosure of personal health information (PHI) to improve the quality of healthcare services provided. While our QPS committee model is designed for quality improvement and safety assessment, some of the experiences and learnings derived from our QPS committee reviews and audits may generate novel data worthy of publication. Though detailed explanation of the ethics review process for data derived from QPS-mediated access to PHI is beyond the scope of this article, the Chair of our local Research Ethics Board is consulted on a case-bycase basis about the type of ethics review indicated when disseminating findings external to our centre.

\section{Incident reports and QPS committee actions}

In the first five years of our QPS committee's existence, over 1,500 PSLS reports were reviewed. The PSLS cases
Canadian Anesthesia Incident Reporting System (CAIRS) to enter QPS committee review process

with similar themes or underlying characteristics are grouped into "clusters". Clustering the reports enables our QPS committee to longitudinally follow the reported frequency of particular event types. Through regular review of incoming reports combined with maintenance of the "cluster" list, the threats that commonly arise in our organization have become more apparent and have helped to clarify our committee's priorities.

Our early experience showed that patient positioningrelated issues were reported frequently and comprised over $25 \%(110 / 415)$ of all submitted PSLS reports over the first two years of our committee's existence. In response to this cluster of positioning events, a multidisciplinary sub-group was convened, led by an existing QPS committee member. This "positioning taskforce" met regularly and reviewed positioning-related reports, sought feedback from the multidisciplinary stakeholders involved in perioperative patient positioning, and initiated a multi-pronged approach to follow and address these issues in an ongoing, iterative fashion. In addition to creating an awareness/education campaign consisting of specific positioning guidelines for staff and trainees, the taskforce's analyses of the events catalyzed creation of a charter of responsibilities to clarify perioperative team members' roles and accountability in the shared activity of patient positioning. Further audit and data collection related to positioning equipment failure (e.g., operating room [OR] bed brackets) resulted in successful purchase requests for new and standardized 
brackets in all ORs (at a time when there was no available budget for new equipment).

Our response to the positioning reports are similar to the comprehensive unit-based safety program (CUSP) approach described by colleagues at $\mathrm{TOH}^{9}$ and elsewhere ${ }^{10}$ to address safety issues in perioperative care. Of interest, the early work of the positioning taskforce was associated with a doubling of position-related PSLS reports, an effect which was sustained over eight months. Perioperative providers at our centre were informed that PSLS reports related to patient positioning would be reviewed by the positioning taskforce. One possibility is that patient safety culture and staff reporting culture changed in response to the newly formed positioning task force. Though voluntary reporting systems are limited in their ability to track the true incidence of events in a system, it is possible that fewer positioning reports represent fewer positioning incidents occurring over time since introduction of the positioning task force. Unfortunately, our data source, as described above, does not permit us to support or refute causation for the change in reporting of positioning events at our centre.

Other clusters noted by our committee included issues related to equipment, drug error, anticoagulation, patient identity, communication, intravenous access, and perioperative support. The largest clusters with the most PSLS reports are shown in Fig. 2. Several actions related to the PSLS reports and clustered events have been taken by the committee with further details of case specifics and responses available for review. ${ }^{11-15}$ Given the multitude of issues, range of reports reviewed, and actions taken, summaries of some of the QPS committee's undertakings are provided in Tables 1 and 2.

\section{Obtain relevant structure, process, and outcome data when possible}

Another important role of a QPS committee is to assess the local data available to drive quality and safety improvement initiatives at either the departmental or hospital level (or both). For example, an ever-growing number of Canadian (and international) hospitals continue to join the National Surgical Quality Improvement Program (NSQIP) from the American College of Surgeons. ${ }^{16}$ This influential program provides hospitallevel performance feedback on multiple, risk-adjusted, 30day outcomes for a wide range of perioperative patients. Programs such as NSQIP can give QPS committees a sense of local perioperative outcome performance, including areas of success and areas that need improvement, relative to other peer hospitals. Should relevant data not be available at one's hospital, a QPS committee may be able to request infrastructure and resources to procure appropriate data sources in the name of quality and safety. Moreover, the outcome data provided from NSQIP may identify areas for improvement at one's centre and provide focus for newly minted CUSP teams in search of issues to address.

Some centres may have their own data available for quality assurance and improvement. The Anesthesia Information Management System (AIMS) at $\mathrm{TOH}$

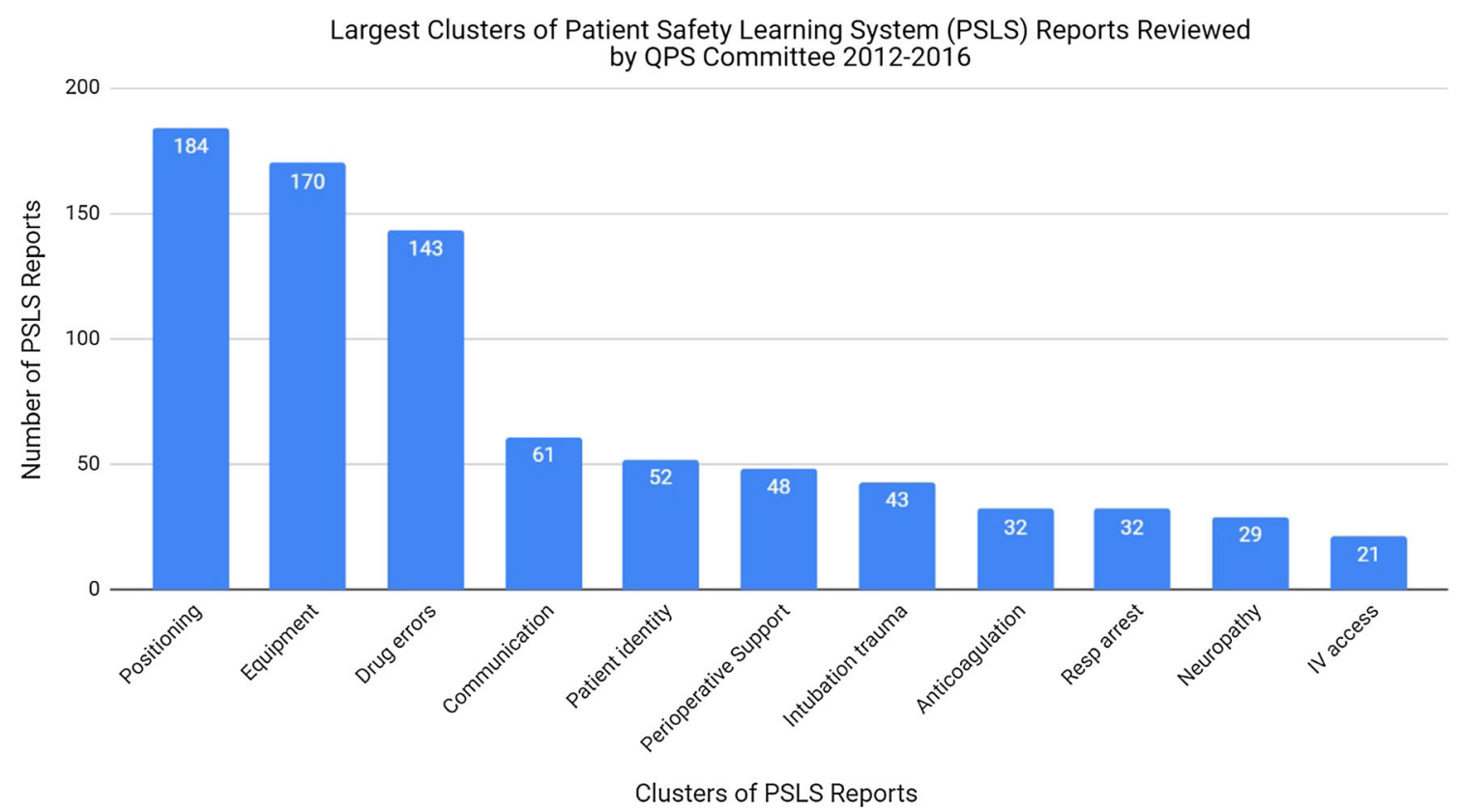

Fig. 2 Largest clusters of patient safety learning system reports reviewed by the quality and patient safety committee 2012-2016 
Table 1 QPS committee undertakings related to medication and equipment over first five years

\section{Medication}

Heparin/mannitol fluid bag supplier changed for look-a-like risk

Epidural and anticoagulation education for ward nurses and non-anesthesiology MDs

Change to anticoagulation orders for hip fracture pathway

Anesthesia drug drawer redesign

Changed sufentanil and "sublimaze"/fentanyl labelling in automated dispensing cabinet

Epinephrine $1 \mathrm{mg} / 1 \mathrm{~mL}$ ampoule removed from regular access location in low-use areas

Succinylcholine available in remote locations

Education surrounding preoperative pregabalin indications

Acute pain service (APS)

- Remove default continuous infusion of opioids for APS electronic medical record (EMR)

- Maximum dose limit added for tramadol on APS EMR

- Standardize SC/PO opioid ordering location on APS EMR

Muscle relaxation monitoring added to Annual Physician Performance Report

Lidocaine IV bags no longer in main OR drug room

Reviewed amount/availability of sugammadex on difficult airway carts and remote locations

Post-partum hemorrhage kit in main OR at all campuses

Process for checking expiration dates of medications on anesthesia drug cart

Remove dextrose fluids from main OR warmers

Calcium replacement standardization

Adenosine available in birthing unit

Add tranexamic acid to anesthesia drug cart*

\section{Equipment}

Patient positioning task force*

- Bed bracket assessment and replacement

- New equipment education protocol and sign off procedure

- Creation of positioning equipment inventory including serial numbers

$25 \mathrm{G}$ cutting spinal needles removed from stock for look-a-like risk with non-cutting needle

Hemorrhage kit available in main OR

Gauze bite block on emergence recommendation

Anaphylaxis kit in main OR at all campuses*

Anesthesia information management system precautions for:

- Emergence agitation

- Parkinson's on treatment

- Cytotoxic medications

Long 22G spinal needles available

Reporting equipment failures with Biomed number

Label on anesthesia machine power cord to prevent unintended removal

Reinforce power cable to vital sign monitor on anesthesia machines

Warning re use of clear-link Baxter IV lock

Review central line kit contents, draping

Line isolation monitor in endoscopy

Remove $50 \mathrm{~mL}$ NS bags for look-a-like risk with $100 \mathrm{~mL}$

Manual NIBP cuffs in anesthesiology care-provided locations

Location of suction tubing, standardization

Review of prone positioning equipment at ambulatory surgical campus

*Denotes trainee-involved projects. IV = intravenous; NS = normal saline; NIBP = non-invasive blood pressure; OR = operating room; $\mathrm{QPS}=$

Quality and patient safety; SC/PO = subcutaneous/per oral 
Table 2 QPS committee undertakings related to processes of care over first five years

Difficult intubation

- Flag available on hospital-wide EMR

- Anesthesia difficult airway report document template on EMR Intraoperative handover checklist*

Handover documentation template on EMR*

Temperature monitoring when using dual modality, active warming technologies

Volatile anesthesia in intensive care unit (ICU) for status asthmaticus/epilepticus guidelines

Eye pain management protocol for non-ophthalmology patients in PACU and ward

Patient positioning taskforce*

- Positioning awareness campaign

- Creation of patient positioning guidelines

- Creation of patient positioning charter of responsibilities

- Creation of patient positioning workshop for new residents

- Bed bracket analysis and replacement

- New equipment education protocol/sign off procedure

- Creation of positioning equipment inventory

Reporting guidelines for PSLS submissions

Critical incident management guidelines for department

Debriefing guide for perioperative events

Anesthesia workstation hygiene

- OR attendant and anesthesia resident education

Advance directive perioperative management and audit*

Anesthesia STAT paging process standardized across campuses

Guidelines for patient complaints related to department employees, trainees, and physicians

Process for patient relations feedback through QPS committee

Preparation of temperature probes for cleaning

Dental trauma management guidelines

Change to surgical safety checklist

- Local anesthesia prompt

- Anticoagulation and neuraxial prompt

- Addition to debrief: reapplication of identification (ID)/allergy bands

- Patient positioning plan and positioning verification

Terms of reference for residents on QPS committee

Modification of QPS document labelling to designate exclusive use for quality improvement

Revision to open tracheostomy process for intubated patients

Adverse dural puncture protocol*

Cuff manometer audit and survey*

Cardiac device management algorithm and guidelines for non-elective cases

Standardize storage of videolaryngoscope stylets

Quarterly meetings with surgeons to discuss shared challenges

Reporting complications in APS electronic medical record

Reviewed/educated staff on proper electrocautery return pad handling/storage

OR cancellation guidelines

Electroconvulsive therapy procedure documentation form reviewed and updated

Requirement for ID and allergy bands for diagnostic imaging patients requiring anesthesiology care

*Denotes trainee-involved projects. EMR = electronic medical record; ICU = intensive care unit; OR = operating room; PACU = postanesthesia care unit; PSLS = patient safety learning system; QPS = quality and patient safety 
captures pre-, intra-, and postoperative data for each patient's perioperative course. In coordination with TOH's performance measurement team, AIMS data are utilized by the committee to produce an annual performance report for each staff anesthesiologist. ${ }^{17}$ This report, provided in advance of each anesthesiologist's annual reappointment interview with the department site head, contains perioperative clinical care process and outcome data for each anesthesiologist and the department as whole. Examples of some of the data points on the performance report include a breakdown of surgical cases completed, mode of anesthesia provided, patient's age, patient's American Society of Anesthesiologists' Physical Status, proportion of patients given timely preoperative antibiotics when indicated, pain scores reported by patients in postanesthesia care unit (PACU), use of neuromuscular blockade monitoring during paralysis, and the number of patients experiencing postoperative vomiting (POV) in the PACU.

When surveyed, two-thirds of our staff anesthesiologists "agreed" or "strongly agreed" that the annual performance report "influenced practice" and "aided in professional development." 17 It is of interest to note that since introduction of the annual performance report, the rate of patients experiencing POV has continually decreased each year. ${ }^{18}$ The reduction in POV was associated with a $20 \%$ relative increase in the proportion of patients given perioperative dexamethasone at our institution (Fig. 3). ${ }^{18}$ Given the quality improvement nature of the "real world" clinical data used to produce our annual performance reports, causation of the reduction in POV cannot be attributed to a specific intervention. It is important to note

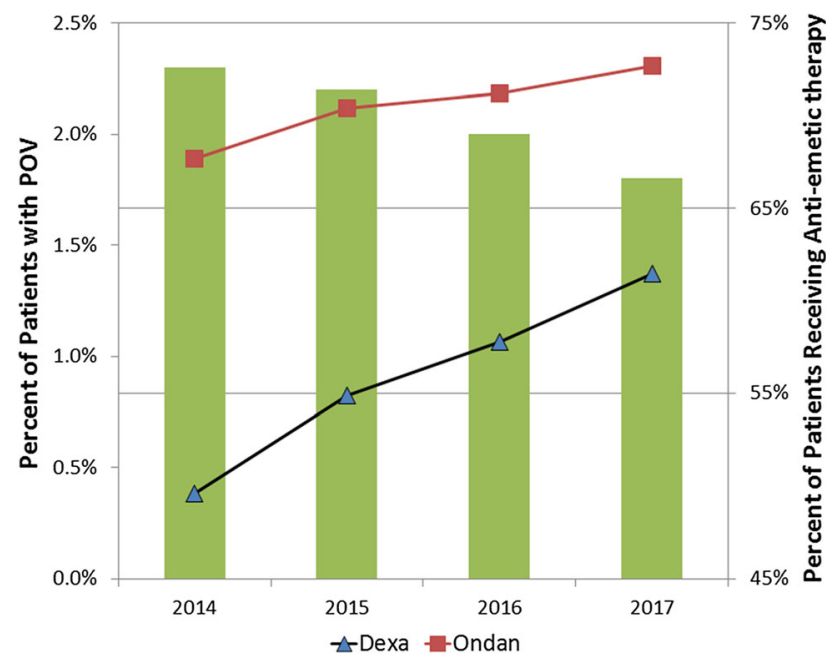

Fig. 3 Percent of main operating room patients with postoperative vomiting $(\mathrm{POV})$ and treatment given over four years at an academic health sciences centre. The POV rates were significantly different between 2014 and 2017 (Chi square, $P<0.001$ ). Reproduced with permission from Pysyk et al. ${ }^{18}$ that the ability to prove causation of changes observed in a clinical system is not the driving principal for use of the clinical data available at our centre. Our philosophy and approach to the data available at our centre is echoed by the CAS Guidelines, ${ }^{5}$ which support use of clinical data from a variety of sources for "monitoring quality of care" via "clinical indicators." In our system, which provides feedback of clinical outcomes to staff anesthesiologists, "the needle moved" in a positive direction for our patients.

\section{Multidisciplinary membership of the QPS committee}

Just as clinical care is multidisciplinary, so too should be the membership of a QPS committee. Coordination of allied healthcare professionals' activities is essential for safe delivery of the complicated process that is perioperative patient care. From the outset, our QPS committee identified that multiple perspectives would be required to facilitate review and discussion of the diverse matters encountered in our perioperative domain. The multidisciplinary composition of the QPS committee provides as complete a perspective as possible of the QPS experience across our perioperative continuum.

Our current QPS committee membership is diverse and includes representatives from the PACU, OR nursing staff, OR management, AART, anesthesiology residents, anesthesiology staff from each clinical campus at TOH including clinical site department heads, pharmacy, patient relations, and $a d$ hoc staff surgeons. Below we explain how the committee evolved to include these perioperative members.

As our department provides clinical care at two separate inpatient campuses and one ambulatory day surgery campus, obtaining site-specific input on the committee was deemed essential for success and buy-in of committee validity amongst colleagues. Many of the issues and PSLS reports identify matters that require inspection and review through a campus-specific lens. Such insight permits appropriate interpretation of similarities and differences of patient profiles and perioperative care practices amongst the campuses. The QPS committee embraces these differences and uses the poly-campus representation to augment discussion of the site-specific issues that arise and seeks to identify common themes that may be applicable to care at other campuses as well.

Medication-related PSLS reports continued to grow in number over the first year of our committee's existence. It was decided that pharmacy representation was essential for our committee to appropriately address the medication issues identified. Indeed, management and response to several of the medication items listed and managed in 
Table 1 were greatly facilitated by pharmacists' input into our QPS committee.

Challenges obtaining patient feedback specific to anesthesia care exist for a multitude of reasons. Unlike surgical colleagues with follow-up in a dedicated, postoperative clinic, opportunities for patient feedback of their anesthesia experience are limited and not part of the care model offered by most anesthesiology departments. To seek an additional mode of feedback on quality of care provided, we approached the Department of Patient Relations to provide a representative for our QPS committee. Patient relations representation on the committee provides unique presentation of the perioperative concerns identified by the patient. This patient-centred approach works in a different manner than the healthcare provider-driven PSLS reports or outcome data (e.g., NSQIP reports) used by our QPS committee to assess the system of care a patient received. Moreover, use of the patient experience to drive change is a central tenet of high-value care. ${ }^{19}$

At a functional level, inclusion of patient relations representation on the QPS committee has facilitated a unique dialogue and sharing of the care experience, between patient and provider, for our department. Though not required for all incidents reported into the PSLS, events identified as critical (leading to death or loss of limb/organ) or serious (leading to major injury resulting in irreversible damage) are automatically directed to a member of the Department of Patient Relations at TOH to facilitate family/patient contact and disclosure of events, if not already performed.

As QPS activities become more integrated with routine clinical department processes, trainee involvement and education are key considerations for QPS committees, particularly in academic centres given the emphasis of "quality improvement" and "patient safety" in the Physician Competency Framework produced by the Royal College of Physician and Surgeons of Canada. ${ }^{20}$ From lecture and learning sessions designed for first-year residents to QPS committee membership, residents of all levels of training and fellows have been involved with our committee's function over the years (Tables 1 and 2). When surveyed, the majority of residents involved with our QPS committee "agreed" or "strongly agreed" that their clinical management of patients had changed as a result of their QPS exposure (unpublished results). These residents also found QPS committee involvement useful for their education and would encourage other residents to become involved in the QPS committee. As trainees interact with "the system" and patients in a somewhat different context than other professionals, the insight and perspective provided by residents and fellows has been enlightening for the committee as a whole.

\section{Conclusions}

This narrative review outlined how perioperative quality of care and patient safety matters have been coordinated by a QPS committee in a Canadian, multi-campus, tertiary care department of anesthesiology and pain medicine. Modification of the QPS committee's membership, to ensure appropriate perioperative stakeholder involvement, has been a key step in permitting timely, effective review of the issues identified at our centre. In particular, involvement of our residents, pharmacy colleagues, and patient relations team members has provided unique perspectives and solutions to the challenges identified.

Two main data sources are regularly reviewed by the QPS committee to set priorities and establish a relevant focus for future QPS initiatives. The first information stream derives from our institution's incident reporting system. Over 1,500 reports have been reviewed in the first five years of our QPS committee's existence. The largest clusters of perioperative events assessed by the QPS committee involved patient positioning, equipment, drug error, anticoagulation, patient identity, communication, intravenous access, and perioperative support. Regular incident report review and upkeep of the cluster list provides a unique lens for evaluation of the issues over time. When the number of cases or rate of cases reported into a particular cluster reaches a critical value, the QPS committee is empowered to take action, such as creation of the patient positioning taskforce.

The second source of information considered by the committee involves institutional clinical care data. Using a combination of local perioperative structure, process, and outcome data, our QPS committee produces an annual performance report for staff anesthesiologists at our centre. Reduced POV associated with a change in antiemetic administration practice has been observed over the years that an annual performance report has been made available to our staff anesthesiologists. ${ }^{18}$

In summary, our five-year experience outlines proof-ofconcept using a QPS committee in an academic department of anesthesiology. The QPS committee provides a platform where relevant perioperative providers meet regularly to enable a department to review events and, when appropriate, institute changes, and/or message and educate peers about emerging patient safety and quality of care threats identified locally. With the advent of CAIRS, ${ }^{6}$ reports entered into the CAIRS system from one's centre may possibly be reviewed by the local department from which the report originated. The structure and processes used by our QPS committee, as highlighted in this article, provide one possible schema for consideration by other departments embarking on their own QPS journey. 
Funding Each author has received support for Non-Clinical Time related to QPS committee activities from the Department of Anesthesiology and Pain Medicine at The Ottawa Hospital.

Conflict of interest None of the authors have any commercial or non-commercial affiliations or conflicts of interest related to the manuscript. None of the authors have received consultancies related to the opinions or matters presented in the manuscript.

Author Contributions All authors contributed to all aspects of this manuscript, including study conception and design; acquisition, analysis, and interpretation of data; and drafting the article.

Editorial responsibility This submission was handled by Dr. Hilary P. Grocott, Editor-in-Chief, Canadian Journal of Anesthesia.

\section{References}

1. Boudreaux AM, Vetter TR. The creation and impact of a dedicated section on quality and patient safety in a clinical academic department. Acad Med 2013; 88: 173-88.

2. Mathews SC, Demski R, Hooper JE, et al. A model for the departmental quality management infrastructure within an academic health system. Acad Med 2017; 92: 608-13.

3. Pronovost PJ, Marsteller JA. Creating a fractal-based quality management infrastructure. J Health Organ Manag 2014; 28 : 576-86.

4. Wang MC, Hyun JK, Harrison M, Shortell SM, Fraser I. Redesigning health systems for quality: lessons from emerging practices. Jt Comm J Qual Patient Saf 2006; 32: 599-611.

5. Dobson G, Chong M, Chow L, et al. Guidelines to the practice of anesthesia - revised edition 2018. Can J Anesth 2018; 65: 76-104.

6. Beattie WS, Culwick MD, Grocott HP. Canadian Anesthesia Incident Reporting System (CAIRS): the Canadian Anesthesiologists' Society's National Patient Safety Initiative. Can J Anesth 2018; 65: 749-56.

7. Kwok ES, Calder LA, Barlow-Krelina E, et al. Implementation of a structured hospital-wide morbidity and mortality rounds model. BMJ Qual Saf 2017; 26: 439-48.
8. Government of Ontario. Personal Health Information Protection Act, 2004, S.O. 2004, chapter 3, Schedule A. Available from URL: https://www.ontario.ca/laws/statute/04p03\#BK44 (accessed July 2019).

9. Moloo H, Brooke R, Sundaresan S, et al. Enabling front linedriven perioperative quality improvement through organizational infrastructure built around the Comprehensive Unit Based Safety Program. Can J Surg 2016; 59: 422-4.

10. Wick EC, Hobson DB, Bennett JL, et al. Implementation of a surgical comprehensive unit-based safety program to reduce surgical site infections. J Am Coll Surg 2012; 215: 193-200.

11. Belcaid I, Poulton A, Pysyk CL. Documentation of perioperative resuscitation status for non-elective surgical patients. J Clin Anesth 2018; 49: 71-2.

12. Miao I, Jee R, Pysyk CL. Under pressure: an audit of airway device pressures. Can J Anesth 2018; 65: 726-7.

13. Pysyk $C L$. A change to the surgical safety checklist to reduce patient identification errors. Can J Anesth 2018; 65: 219-20.

14. Pysyk CL. Risk of look-a-like equipment in anesthesiology: it's not just medications. Can J Anesth 2017; 64: 1291-2.

15. Pysyk CL, Abdulla KR, Boet $S$. Goldilocks and the perioperative patient: not too hot, not too cold. Acta Anaesthesiol Scand 2017; 61: 460-1.

16. American College of Surgeons. National Surgical Quality Improvement Program (NSQIP), American College of Surgeons. Available from URL: https://www.facs.org/qualityprograms/acs-nsqip (accessed July 2019).

17. Wheeler K, Baxter A, Boet S, Pysyk C, Bryson GL. Performance feedback in anesthesia: a post-implementation survey. Can J Anesth 2017; 64: 681-2.

18. Pysyk CL, Corsi DJ, Boet $S$. The potential for anesthesiologist practice feedback to reduce postoperative vomiting in an academic centre. Can J Anesth 2019; 66: 609-10.

19. Berwick DM, Nolan TW, Whittington J. The triple aim: care, health, and cost. Health Aff (Millwood) 2008; 27: 759-69.

20. Frank JR, Snell L, Sherbino J. CanMEDS 2015 Physician Competency Framework. Ottawa: Royal College of Physicians and Surgeons of Canada; 2015. Available from URL: http://www. royalcollege.ca/rcsite/documents/canmeds/canmeds-full-frameworke.pdf (accessed July 2019).

Publisher's Note Springer Nature remains neutral with regard to jurisdictional claims in published maps and institutional affiliations. 